\title{
Scientific evidence of the solution to the problem of compacting man-made sandy sub-structures in winter conditions
}

\author{
Oleg Minaev ${ }^{1, *}$ \\ ${ }^{1}$ Admiral Makarov State University of Maritime and Inland Shipping, 5/7, Dvinskaya str, Saint- \\ Petersburg, 198035, Russia
}

\begin{abstract}
In the recent time the issue of erecting soil sub-structures and constructions at negative temperatures in winter conditions and the areas of permafrost has been really important, so such research studies are very topical. The paper provides for the results of experimental compacting of medium sands of a sub-structure in winter conditions (at the ambient temperature of $-20^{0} \ldots 25^{0} \mathrm{C}$ ) in the construction of a complex of protective structures (CPS) preventing floods in St. Petersburg. Heavy and mediumheavy vibratory compactors were used in the experimental sections. Before compaction, sandy soils of the sub-structure with the layer thickness equal to $0.4 \ldots 0.5 \mathrm{~m}$ were placed. For comparison purposes, the paper contains the results of layer-by-layer compaction of such sands in the summer season with application of vibratory compactors and deep-water vibratory plant in the subs-structure of the freeway along the ridge of the protective dam from the side of the Gulf of Finland in the construction of the CPS.
\end{abstract}

\section{Introduction}

In the recent time the issue of erecting soil sub-structures and constructions at negative temperatures in winter conditions and in the areas of permafrost has been really important, so such research studies are very topical [1].

Today there are two main principles established by Prof. N.A. Tsytovich for using frozen soils as sub-structures of buildings and constructions. According to the second principle, the most reasonable and promising is preliminary thawing of frozen soils and their further compaction. However, there is no data in literature about the density of compaction of soils in winter conditions with application of vibratory compactors and the vibratory compaction of the former after thawing.

According to the research studies of some scientists, including those of the author of this article, bound water on the surface of solid particles in wet soil results in their parting in relation to each other. The so-called "wedging effect" increases the distance between neighboring particles of soil (Fig.1).

\footnotetext{
* Corresponding author: minaev.op@bk.ru
} 
According to Prof. P.L. Ivanov, due to this effect the porosity factor $e$ of sandy soils with a diverse granulometric composition grows by approximately 2 times in the humidity ramp of $4-8 \%$.

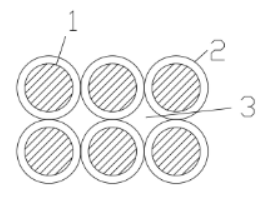

Fig. 1. The scheme of the wedging effect of bound water: 1-soil particle, 2 - bound water film, in winter conditions-ice, 3 - free gas in soil pores.

The author's own research has shown (see Fig. 2) that during humidification of dry ash due to the forces of electro-molecular interaction of water and ash particles, the ash swells considerably in volume by 1.2-2 times. At the same time wet soils get compacted considerably after different dynamic impacts (vibration, impulse).

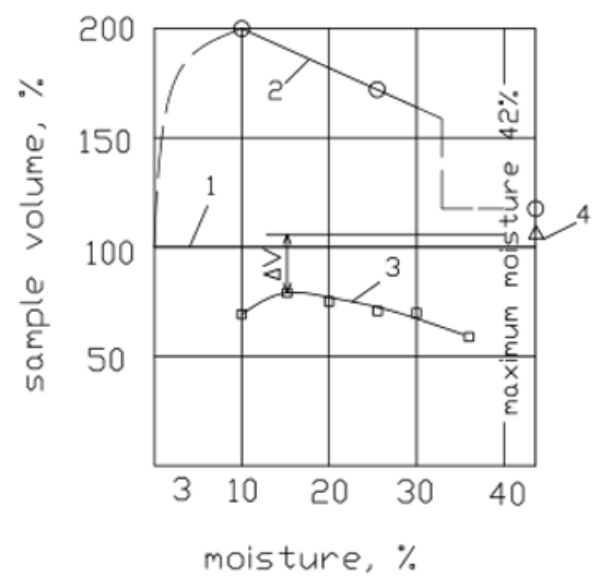

Fig. 2. The results of the laboratory experiments aimed at researching the change in the volume of ash after humidification and dynamic compaction: 1 - the volume of dry material sample, 2 and 3 respectively - after its humidification and dynamic compaction, 4 - as a result of complete wetting and free squeezing of water without a load.

The paper provides for the results of the experimental compacting of medium sands in winter conditions (at the ambient temperature of $-20^{\circ} \ldots 25^{\circ} \mathrm{C}$ ) in the construction of a complex of protective structures (CPS) preventing floods in St. Petersburg. Heavy and medium-heavy vibratory compactors were used in the experimental sections. For comparison purposes, the paper contains the results of layer-by-layer compaction of such sands in the summer season with application of the same vibratory compactors and a deep-water vibratory plant in the subs-structure of the freeway along the ridge of the protective dam from the side of the Gulf of Finland in the construction of the CPS.

\section{Theoretical justification}

According to the research by Prof. O.A. Savinov and Prof. I.I. Blekhman [3], the best compaction for loose medium occurs when

$$
\mathrm{A} \omega^{2}=(0.9-1) g
$$


where $\mathrm{A}$ is the vibration amplitude; $\omega$ is circular frequency of vibrations; $g$ is gravitational acceleration.

It is known [4] that in viscous media the vibration amplitude decreases with distance $\mathrm{x}$ from the vibration source exponentially:

$$
A_{r}=A_{0} e^{-\frac{r}{\delta}}
$$

where $A_{r}$ is the critical vibration amplitude, i.e. minimum allowed for full dilution of the soil in the compacted area; $A_{0}$ is the vibration amplitude in the vicinity of the compactor's vibrating element; $r$ is the distance from the vibration source, $\mathrm{m} ; \delta$ is the vibration damping coefficient with the distance from the vibration source, equal to $0.8-0.9$ and $0.3-0.4$ for medium-sized and fine sand respectively, $\mathrm{m}$.

The value of the vibration amplitude of the compactor $A_{o}$ at a given depth of the substructure is determined by the expression:

$$
A_{o}=\frac{P_{o}-P^{d y n}}{m \omega^{2}}=\frac{P_{o}-\left(P_{\mathrm{fr}}^{d y n}+P_{\mathrm{tip}}^{d y n}\right)}{\left(m_{\mathrm{v}}+m_{\mathrm{comp}}\right)(2 \pi f)^{2}},
$$

where $P_{o}$-is the minimum driving force of the vibratory driver, $m_{\mathrm{v}}$ and $m_{\text {comp }}$ are masses of the vibratory driver and the compactor, $f$ - are frequency of the vibration, $P_{\mathrm{fr}}^{d y n}$ and $P_{\text {tip }}^{d y n}$ - are dynamic resistance forces in accordance with friction along the lateral surface of the compactor and its frontal immersion, $\mathrm{kN}$.

As it is known from the research of Prof. M.G. Zeitlin and Prof. V.V. Verstov, and also from the author's research[5], during dynamic driving of piles into water-saturated sandy soils, a decrease in effective resistance of the soil to shift and a temporary decrease in resistance to driving of piles occur due to shaking and redundant pore-water pressure. Particularly, dynamic resistance forces during vibro immersion (extraction) are below the corresponding statistic ones in water-saturated soils by friction along the lateral surface by 4.5-6 times and by frontal immersion by 2.5-3 times.

Using dependences (1)-(3), let us calculate the initial amplitudes and the compacted area by the immersion depth of the compactor of VNIIGS design, which is immersed with the vibratory driver VPP-2, depending on values of friction resistance along the lateral surface of the compactor and frontal resistance to its immersion. The obtained values are the evidence of the fact that, when reaching $6 \mathrm{~m}$ of the compaction depth, the soil compaction area around the "vibro-firtree" extends from 3 to $4.06 \mathrm{~m}$ while the compactor gets closer to the foundation soil surface.

According to the suggestion of the author of this paper, in order to increase the compaction depth, the vibratory plant designed by VNIIGS was modernized with spatial elements being placed only in the lower part of the rod of the compactor. Based on this, similar calculations were carried out for the modernized vibratory plant with a triple reduction of spatial elements in the lower part of the compactor. Let us represent the obtained data as graphs in fig. 3 .

From fig. 3 it is obvious that the soil compaction depth of the vibratory plant of VNIIGS design increases from $6 \mathrm{~m}$ (fig. 3, graph 1) to $9.53 \mathrm{~m}$ (fig. 3, graph 2) when using the improved compactor while the compaction area remains the same and is equal to $3 \mathrm{~m}$ for both types of the compactors and other parameters are constant. 


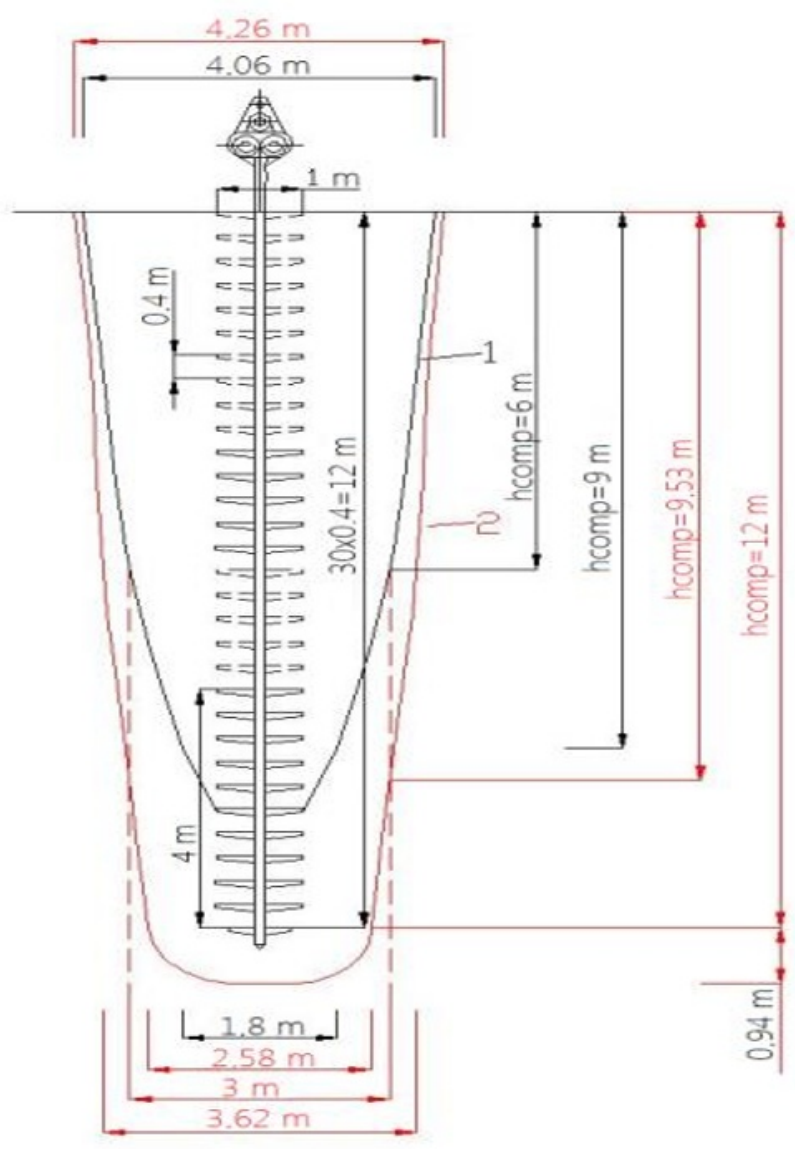

Fig. 3. Graphs of compaction areas of water-saturated sandy foundation soils of medium size to the depth of $12 \mathrm{~m}$ with the vibratory plant of improved design (1), and of decrease in the soil compaction area and in immersion depth of the compactor of VNIIGS design (2).

Besides, the improved compactor allows increasing the compaction depth of the foundation to $12 \mathrm{~m}$ and deeper. Thus, the compaction area, obtained by calculations at the depth of $12 \mathrm{~m}$, is $2.58 \mathrm{~m}$ (see fig. 3, graph 2) and can be considered fully acceptable for effective use of the vibratory plant at the given soil compaction depth.

Similar calculations for the plant designed at VNIIGS show that the diameter of the compaction area at the depth of $9 \mathrm{~m}$ decreases to $1.8 \mathrm{~m}$, which is less than the required (according to VNIIGS) minimum limit of $2 \mathrm{~m}$ (see fig. 3, graph 1). And at the same time, the compactor of this design cannot be immersed to the depth of $12 \mathrm{~m}$ at all.

\section{Experiments}

The experimental placement of sandy soils with application of vibratory compactors was carried out in the construction of the complex of protective structures (CPS) of St. Petersburg to prevent floods. At the time of the experimental work the temperature of ambient air was minus $20 \ldots 25^{\circ} \mathrm{C}$. Sandy soils were supplied to the experimental site from the in-washed stock pile in the summer season. The granulometric composition of the sands, laid into the dam body, is shown in fig. 4. 


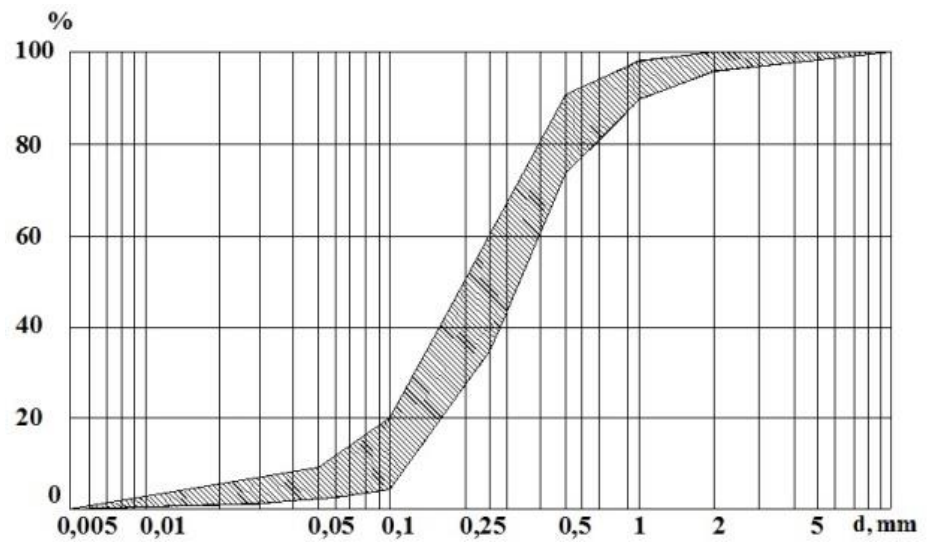

Fig. 4. Granulometric composition of the sandy soil laid into the dam body according to data of selecting soil samples from bored wells.

Heavy and medium-heavy vibratory compactors were used in the experimental section. Surface leveling of the placed soil with the layer thickness of $0.4 \ldots 0.5 \mathrm{~m}$ was carried out for compaction. The soil in the experimental sections was compacted with 6-8 drivings of a compactor along one trace.

Soil samples taken after vibration compaction in the experimental sections showed that the density of the soil skeleton $\rho d$ was $1.48 \ldots 1.52 \mathrm{~g} / \mathrm{cm} 3$. This meant that when sandy soils are dumped in winter conditions they are placed with the soil skeleton density $\rho d$ being "looser than loose", i.e. being less than the density of soil in the maximally loose composition.

Consequently, at negative temperatures of the ambient air, water on the surface of solid particles freezes and turns into ice. It can be suggested that when frozen soil particles rub against each other during intensive vibration, iced water films get heated and soil gets compacted to some extent as they are getting thawed.

We should also note that the existing experience in compacting such soils at the natural humidity of $4-6 \%$ in summer conditions with the same vibratory compactors proved that the obtained density of soil skeleton $\rho_{d}$, equaled to $1.57 \ldots 1.59 \mathrm{~g} / \mathrm{cm}^{3}$, which was lower than the designed density of soil placed at the construction site of the complex of protective structures (CPS) preventing floods in St. Petersburg.

The conducted research made it possible to take a decision to dump the sandy soil of the body of the dam "in dry", with the thickness of a layer up to $4 \mathrm{~m}$ in winter conditions without compaction. Further compaction of the soils of the body of the dam was meant for the summer period for the entire depth of $9 \mathrm{~m}$ of the placed stratum. At this, the depth of the sub-water dump of the above sands of the body of the dam was $5 \mathrm{~m}$.

For this originally, the vibratory plant of VNIIGS design was initially tested. It included the vibratory driver VSh-1 and a compactor $11.2 \mathrm{~m}$ long made of a heavy-wall pipe $133 \mathrm{~mm}$ in diameter, to which along the whole length horizontal ribs are welded at a distance of $500 \mathrm{~mm}$ from each other. Taking these ribs into account, the diameter of the spatial compactor was $700 \mathrm{~mm}$. To facilitate the immersion of the compactor into the foundation soil, the tubular rod was equipped with a cruciform tip. Water was distributed to the socket with the output of $300 \mathrm{~m}^{3} / \mathrm{h}$ and the pressure of $90 \mathrm{mwc}$ with a possibility of changing these parameters using a flap, which was installed in the water-induction system. A crane with $25 \mathrm{t}$ of carrying capacity was used as a carrier vehicle.

Fine and medium sands of the foundation were experimentally compacted at an earth dam to the mark of about $4 \mathrm{~m}$ above the zero ordnance datum. The depth of underwater dumping of the mentioned sands was $5 \mathrm{~m}$. 
Data of the statistical penetration test showed that the soil on the whole was compacted within a radius up to $3 \mathrm{~m}$ from the center of the compactor to the depth down to $9 \mathrm{~m}$.

Afterwards, the structure of the compactor was modified and new experimental work aimed at compacting the soils of the sub-structure was conducted at the same section of the dam with the use of the modernized vibratory plant.

Comparative field studies of the vibratory plant designed by VNIIGS and the modernized construction were conducted after the structure of the compactor was modified with cutting off the top horizontal ribs and keeping them only in the section of 3.5-4.5 $\mathrm{m}$ in the lower part of the rod of the compactor. A less powerful vibratory driver V-401 was used to submerge the compactor. Moreover, since the vibratory driver V-401 is far more lightweight than the vibratory driver VSh-1, a crane with a much less weight-lifting capacity.

Tests of the improved compactor with the vibratory driver $\mathrm{V}-401$ were conducted at the immersion pitch of $3 \times 3 \mathrm{~m}$.

According to data of the statistical penetration test, the foundation compaction depth was $9 \mathrm{~m}$. At that, more unified soil compaction was reached both in the central area and within a radius of $1.5 \mathrm{~m}$. Besides, the soil was not loosened in the central area around the compactor.

The selection of soil samples with a cutting ring in the spaces between subsidence craters to the depth of $1.5 \mathrm{~m}$ from the foundation surface showed that density of the soil skeleton was $1.62 \mathrm{~g} / \mathrm{sm}^{3}$ with $90 \%$ coverage and $1.69 \mathrm{~g} / \mathrm{sm}^{3}$ with $50 \%$ coverage. No decrease in soil density was registered when moving away from the compactor's immersion center. Taking into account that, according to data of laboratory tests, the mellow density of dry soil was $\rho_{s k}^{\text {loose }}=1.49 \mathrm{~g} / \mathrm{sm}^{3}$ on average and the solid density was $\rho_{s k}^{\text {dence }}=1.81 \mathrm{~g} / \mathrm{sm}^{3}$, using dependency (12) let us determine the relative density $I_{D}$ of the foundation soils which is equal to 0.45 and 0.67 respectively.

According to data of the statistical penetration test, after compaction with the improved plant, resistance to the probe point penetration $q_{3}$ generally increased to 9-14 MPa along the whole depth of the compacted layer of the foundation. The given values of $q_{3}$ correspond with the values of the relative density of foundation soils within the limits of $I_{D}=0.48-0.76$. Also, a considerable augmentation in physical and mechanical characteristics of foundation soils was achieved: in the modulus of elasticity $E$ from $6 \ldots 24 \mathrm{MPa}$ to $27 \ldots 42 \mathrm{MPa}$, in the angle of internal friction $\varphi$ from 26 ...32 to 32-34 degrees.

So, the data by $I_{D}$ characterize fine and medium sands as of medium density and solid ones. It ensures resistance of the structure of sandy foundation soils of buildings to the majority of dynamic, including seismic, impacts [6-13].

Vibratory compactors were used to compact the surface sand-gravel and crushed stone layer to the design elevation ( $6 \mathrm{~m}$ higher than the zero elevation) of the sub-structure of the automobile road. A heavy Russian-made vibratory compactor K $701 \mathrm{M}-\mathrm{VK}$ can be successfully used for this purpose (Fig. 5). This vibratory compactor was designed with the scientific and methodological support of the Russian experts in vibratory machinery under the supervision of Prof. O.A. Savinova [14]. 


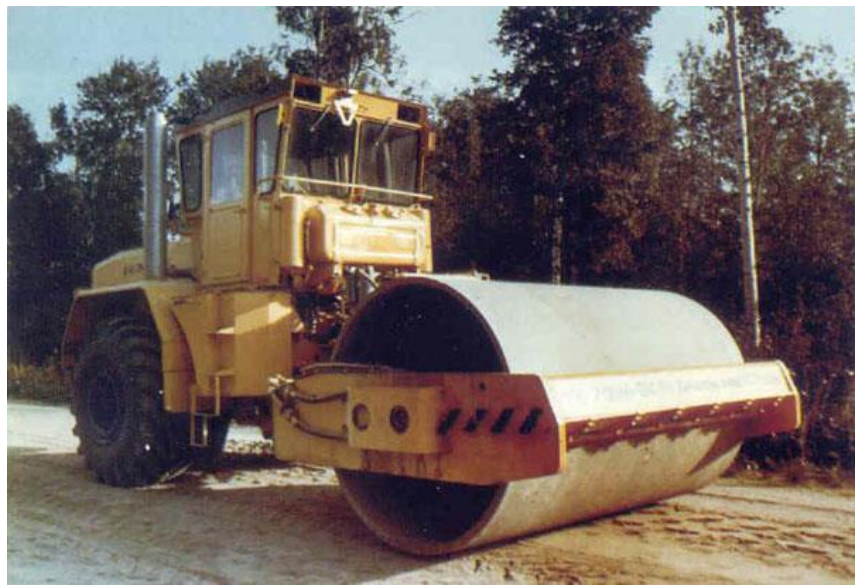

Fig. 5. Vibratory roller K-701 M-VK with the smooth drum.

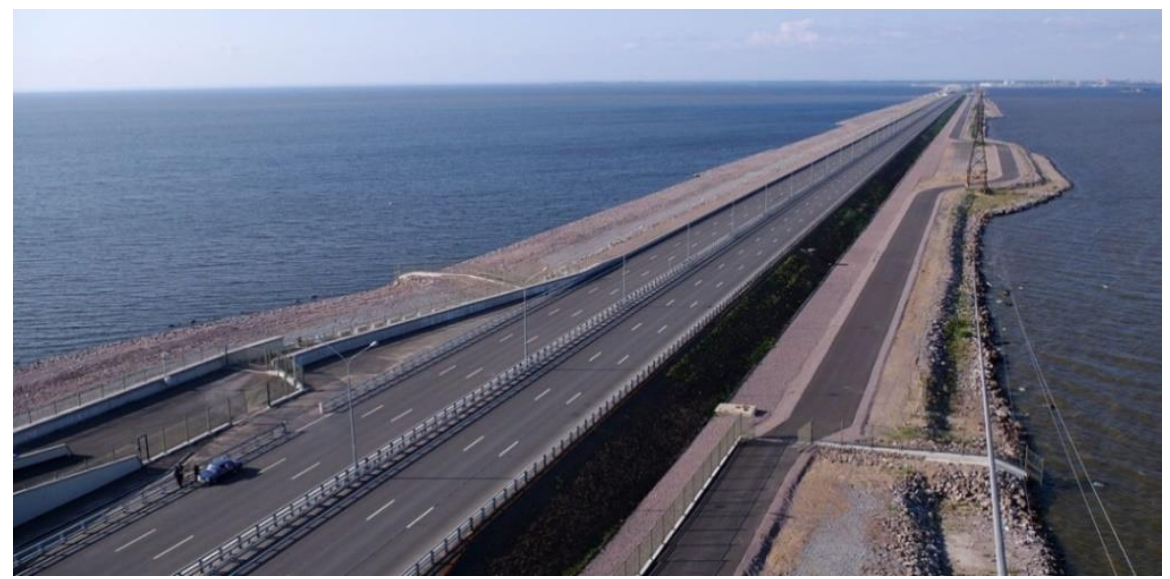

Fig. 6. Present view of the expressway along CPS.

The vibratory plant with the improved compactor, immersed with the vibratory driver VPP-2, was used for compaction of the back of the dam with an expressway (see fig. 6), for compaction of the sandy filling in ballasts of navigable passes and other objects during the construction of a complex of protective structures (CPS) preventing floods in St. Petersburg. The output in different parts of just the CPS construction site was no less than 1 million $\mathrm{m}^{3}$ of the compacted soil. It should be noted that at CPS constructing, it was the first time in the building practice when this vibratory plant was used for the compaction of large bulks (about 300 thousand $\mathrm{m}^{3}$ ) of soil at one construction site.

\section{Conclusions}

1. When sandy soils are dumped in winter conditions they are placed with the soil skeleton density $\rho_{d}$ being "looser than loose", i.e. being less than the density of soil in the maximally loose composition. It means that at negative temperatures of the ambient air, water on the surface of solid particles freezes and turns into ice. In these conditions layer-by-layer compaction of sandy soils by vibratory compactors is not very effective.

2. In winter conditions, it is reasonable to choose in favor of placing sandy soils of the sub-structure without compaction. Further compaction of sands after thawing in summer 
conditions can be carried out for the entire depth of the placed stratum, including in the subwater zone of placement, with application of a deep-water vibratory plant.

3. Heavy Russian-made vibratory compactors K701 M-VK can be used for layer-by-layer compaction of sandy, sandy-gravel and crushed stone soils in the sub-structure of high-speed railroads and freeways.

\section{References}

1. O.P Minaev, A.P. Krutov, Hydrotech. Constr. 27(7), 424-428 (1993)

2. M.Y. Abelev, I.V. Averin, U.A. Korableva, S. Mech. and Found. Eng. 52 (2), 84-87 (2015)

3. A.M. Uzdin, T.A. Belash, I.I. Blekhman, S. Mech. and Found. Eng. 48 (5), 182-189 (2011)

4. O.P. Minaev, Mag. of Civ. Eng. 51(7), 66-73 (2014)

5. O.P. Minaev, S. Mech. and Found. Eng. 22(2), 56-60 (1985)

6. V.A. Florin, P.L Ivanov, Proc. of the Y Int. Conf. on S. Mech. and Found. Eng. 1, 182186 (1961)

7. H.B. Seed, I.M. Idriss, I.M., Ground motions and soil liquefaction during earthquakes (Earthq. Eng. R. Inst., Oakland, 1982)

8. I.M., Idriss, R.W. Boulanger, Soil liquefaction during earthquakes (EERI, USA, 2008)

9. K. Ishihara, Soil Behaviour in Earthquake Geotechnic (Clarendon Press, Oxford, 1996)

10. L.R. Stavnitser, G.A. Nikitaeva, S. Mech. and Found. Eng. 45 (1), 9-12 (2008)

11. I. Towhata, S. Goto, Y. Taguchi, Proc. of the 15th Asian Reg. Conf. on S. Mech. and Geot. Eng., 800-805 (2015)

12. S. Yasuda, K. Ishihara, K., Harada, S. and Found., Iss. Spec., 99-107 (1996)

13. A.Zh. Zhussupbekov, T. Tanaka, A.K. Aldungarova, Proc. of the 15th Asian Reg. Conf. on S. Mech. and Geot. Eng., 1546-1550 (2015)

14. O.P. Minaev, S. Mech. and Found. Eng. 48 (5), 190-195 (2011) 